\title{
Effect of neonatal exposure to the antioestrogens nafoxidine and CI-628 upon the development of the uterus in the prepubertal rat
}

\author{
P. S. Campbell and P. M. Satterfield \\ Department of Biological Sciences, The University of Alabama in Huntsville, Huntsville, AL 35899, \\ U.S.A.
}

\begin{abstract}
Summary. Neonatal Sprague-Dawley rats were injected with the antioestrogens nafoxidine or CI-628 on Day 3 of life alone or in combination with oestradiol benzoate $24 \mathrm{~h}$ later. Oestrogen-stimulated glucose oxidation and cytoplasmic oestrogen binding sites of the uteri were assessed at 21-23 days of age. Neither antioestrogen antagonized the prepubertal uterine impairments produced by neonatal oestradiol treatment. Both antioestrogens administered alone produced deficits which mimicked those produced by neonatal oestrogenization. However, the agonist property of each antioestrogen was differentially expressed: treatment with CI-628 reduced prepubertal oestrogen binding sites in the uterus, but nafoxidine exposure decreased the sensitivity of the uterus to oestradiol stimulation of glucose oxidation. It is postulated that CI-628 directly affects the uterus to reduce production of oestrogen receptor protein, while nafoxidine affects the development of the uterine phosphogluconate oxidative pathway indirectly through impaired ovarian function. However, antioestrogens blocked the neonatal oestradiol-induced reduction in the oestrogen-stimulated production of actomyosin in the adult uterus. Therefore, while both CI-628 and nafoxidine are clearly agonists in the neonatal rat, each appears to exhibit cell-specific agonist and antagonist properties.
\end{abstract}

Keywords: uterus; antioestrogen; oestrogen receptor; glucose metabolism; neonate; rat

\section{Introduction}

It has been previously demonstrated (Gellert et al., 1977; Campbell, 1980) that neonatal administration of oestradiol benzoate results in impaired uterine growth responses to exogenous oestradiol in the prepubertal rat. This reduction in uterine responsivity is the result of a decrease in available cytoplasmic oestrogen binding sites in the uterus with a concomitant decrease in the nuclear retention of the receptor-oestrogen complex by uterine nuclei (Gellert et al., 1977; Campbell, 1980). Additionally, neonatal exposure to oestradiol benzoate impairs the capacity of the uterus to metabolize glucose under oestrogen stimulation (Campbell, 1980). The latter effect augments the severity of disturbance of uterine function by neonatal oestrogenization in contrast to that produced by neonatal androgenization.

The purpose of the experiments reported herein was therefore to determine whether the antioestrogens CI-628 and nafoxidine would allay the effects of neonatal oestrogenization by oestradiol benzoate or whether their agonist properties would prevail over their antagonistic action.

\section{Materials and Methods}

Preparation of animals and materials. Rats of the Sprague-Dawley strain (SAF/SDD) were obtained from Southern Animal Farms (Prattville, AL) and maintained in a controlled environment in the UAH animal facility with a lighting 
schedule of $13 \mathrm{~h}$ light: $11 \mathrm{~h}$ dark (lights on $07: 00 \mathrm{~h}$ ). Commercially pelleted food (Purina) and water were provided $a d$ libitum.

Young were designated 1 day old on the morning of discovery, and litters were culled to a maximum of 8 females with only one treatment group housed with a given mother. All experiments used animals from a minimum of two different litters. Neonates were injected subcutaneously (s.c.) with peanut oil vehicle $(0.1 \mathrm{ml})$, $50 \mu \mathrm{g} \mathrm{CI}-628$ ( $\alpha$ - $\{4$-pyrrolidinoethoxy $\}$-phenyl-4-methoxy- $\alpha$-nitrostilbene) or $50 \mu \mathrm{g}$ nafoxidine (1-2-\{p-(3,4-dihydro6-methoxy-2-phenyl-1-naphthy)phenoxyl\}ethyl pyrrolidine $\mathrm{HCl}$ ) on Day 3 of life. On Day 4 additional injections of $100 \mu \mathrm{g}$ oestradiol benzoate or peanut oil vehicle were administered. Other rats were neonatally ovariectomized on Day 3 of life under hypothermia-induced anaesthesia. Rats were killed at 21-23 days of age by cervical dislocation for measuring uterine cytoplasmic oestrogen binding sites and oestrogenstimulated glucose metabolism. Some neonatally-treated rats were allowed to reach adulthood and between 60 and 80 days of age were bilaterally ovariectomized under ether anaesthesia for subsequent actomyosin determination.

Oestradiol benzoate, diethylstilboestrol (DES), oestradiol-17 $\beta$ and all laboratory chemicals were obtained from Sigma Chemical Co. (St Louis, MO). The antioestrogens nafoxidine and CI-628 were generously provided by the Upjohn Company (Kalamazoo, MI) and Warner-Lambert/Parke-Davis (Detroit, MI), respectively. [6,7- $\left.{ }^{3} \mathrm{H}\right]$ Oestradiol (sp. act. 49-51 Ci/mmol) was obtained from the New England Nuclear Corporation (Boston, MA) and had a radiochemical purity of $98 \%$. D-[(U) $\left.-{ }^{14} \mathrm{C}\right]$ glucose (sp. act. $1 \cdot 19-1 \cdot 92 \mu \mathrm{Ci} / \mu \mathrm{g}$; radiochemical purity $99 \%$ ) was also acquired from NEN. A Beckman LS-100C scintillation counter was used to determine the radioactivity of the samples using Aquasol-2 (NEN) as the scintillation cocktail. Tritium was counted at $38 \%$ efficiency and carbon-14 was counted at $75 \%$ efficiency.

Determination of cytoplasmic oestrogen binding sites. Soluble oestrogen binding sites were measured by the charcoal-dextran assay (Korenman, 1968). Each uterus was quickly removed, stripped of adhering fat and connective tissue and homogenized in $2 \mathrm{ml}$ ice-cold TE buffer (10 mM-Tris-1.5 mM-EDTA, pH 7.4) using a motordriven Kontes Duall glass homogenizer. After centrifugation at $1000 \mathrm{~g}$, the cytosol of each sample was divided into two $0.5 \mathrm{ml}$ samples labelled $A$ and $B$. Tube $A$ received $10^{-8} \mathrm{M}-\left[{ }^{3} \mathrm{H}\right]$ oestradiol while tube $B$ received the labelled oestradiol plus 100-fold excess DES. The assay tubes were incubated for $60 \mathrm{~min}$ at $04^{\circ} \mathrm{C}$ with vortexing every $15 \mathrm{~min}$. At the end of incubation an equal volume of $1.0 \%$ charcoal- $0.05 \%$ dextran suspension was added to adsorb unbound $\left[{ }^{3} \mathrm{H}\right]$ oestradiol and the tubes were incubated for another $15 \mathrm{~min}$. After the charcoal-dextran particles were pelleted, a $200 \mu \mathrm{l}$ sample of supernatant was removed from each tube and added to $10 \mathrm{ml}$ scintillation cocktail to determine specific binding. At least $95 \%$ of the specific oestrogen binding sites in the immature rat uterus are occupied when oestradiol is present in the assay buffer at $10^{-8} \mathrm{M}$ (Anderson et al., 1973).

Oestrogen-stimulated glucose metabolism. The oxidation of $\left[\mathrm{U}-{ }^{14} \mathrm{C}\right]$ glucose to ${ }^{14} \mathrm{CO}_{2}$ was determined by the method of Nicolette \& Gorski (1964). Animals were injected s.c. with $0 \cdot 1 \mu \mathrm{g}$ oestradiol-17 $\beta$ and uteri were obtained $3 \mathrm{~h}$ later. The dose of oestradiol used is the minimal dose required to promote maximum stimulation of glucose oxidation for this time interval (Anderson et al., 1973). The uteri were stripped of adhering connective tissue and placed into $25 \mathrm{ml}$ Erlenmeyer flasks containing $2 \mathrm{ml}$ Eagles medium (Difco; Detroit, MI) and $10^{-6} \mathrm{M}-\left[\mathrm{U}-{ }^{14} \mathrm{C}\right] \mathrm{glucose}$ $\left(0.5 \mu \mathrm{Ci}\right.$ total activity). The flasks were aerated with $95 \% \mathrm{O}_{2}-5 \% \mathrm{CO}_{2}$ and sealed with rubber serum stoppers containing filter paper wicks soaked with $0.25 \mathrm{ml}$ methyl benzonium hydroxide to trap evolved ${ }^{14} \mathrm{CO}_{2}$. The flasks were incubated $1 \mathrm{~h}$ at $37^{\circ} \mathrm{C}$ after which $1.5 \mathrm{ml} 5 \mathrm{~N}-\mathrm{H}_{2} \mathrm{SO}_{4}$ was injected through the serum stoppers to halt the reaction and promote the release of carbon dioxide from the incubation medium. After an additional $2 \mathrm{~h}$ incubation at $37^{\circ} \mathrm{C}$, the ${ }^{14} \mathrm{CO}_{2}$ which had been absorbed onto the filter paper wick was determined by placing the wick into $10 \mathrm{ml}$ Aquasol-2 and counting the radioactivity.

Determination of actomyosin. Ovariectomized adult rats were injected s.c. for 6 consecutive days with $5 \mu \mathrm{g}$ oestradiol- $17 \beta / 100 \mathrm{~g}$ body weight. Uteri were obtained $24 \mathrm{~h}$ after the last injection and kept frozen at $-20^{\circ} \mathrm{C}$ until the actomyosin content could be analysed. The extraction and estimation of actomyosin was carried out according to the procedure described by Needham \& Williams (1963). To quantitate actomyosin content, 2 or 3 uteri from the same treatment group were placed into an ice-cold solution containing three times the uterine volume of $0.5 \mathrm{M}-\mathrm{KCl}$ buffered with $0.04 \mathrm{M}-\mathrm{K}_{2} \mathrm{HPO}_{4}(\mathrm{pH} 7 \cdot 1)$ and $1 / 50$ of the volume of $0.1 \mathrm{M}$-ATP adjusted to pH 7.4 with Trizma base. The uteri were homogenized using a Polytron homogenizer and the homogenate was centrifuged at $1000 \mathrm{~g}$ for $10 \mathrm{~min}$ at $4^{\circ} \mathrm{C}$. The supernatant was decanted and reserved for later actomyosin estimation. The pellet was resuspended at two times the uterine volume with the buffered $\mathrm{KCl}$ solution and then centrifuged again for $10 \mathrm{~min}$. The two supernatant pools were combined and stored overnight at $4^{\circ} \mathrm{C}$ to permit ATP degradation.

Viscosity measurements were made the next day using an Ostwald viscosimeter to estimate actomyosin content of the uteri. Uterine supernatant was added to the viscosimeter and flow rates of the supernatant between two fiducial marks were determined at $25^{\circ} \mathrm{C}$ in the presence or absence of $0.1 \mathrm{M}-\mathrm{ATP}-\mathrm{MgCl}_{2}$. The concentration of actomyosin in each uterine fraction was then calculated on the basis of the difference in the two viscosities determined in the presence or absence of ATP according to an equation described by Needham \& Williams (1963).

Total uterine protein was measured by the Folin-Ciocalteau method described by Lowry et al. (1951) using crystalline bovine serum albumin as the standard. 


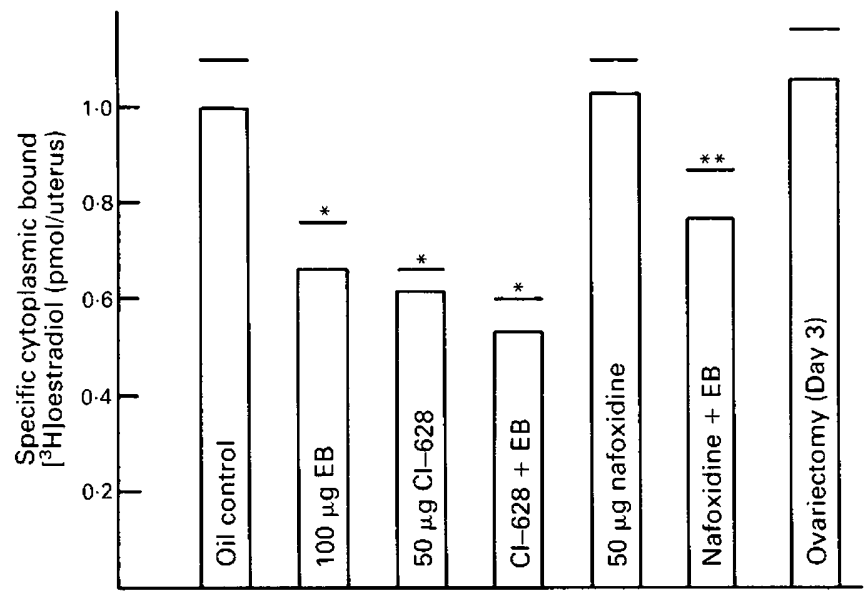

Fig. 1. Cytoplasmic oestrogen binding sites in uteri of prepubertal rats neonatally treated with oestradiol benzoate (EB), antioestrogen (CI-628 or nafoxidine) or ovariectomized. Each value is the mean \pm s.e.m. of 8 different determinations. All treatments were compared to the vehicleinjected controls by Dunnett's T statistics. ${ }^{*} P<0.025 ;{ }^{* *} P<0.05$.

\section{Results}

As illustrated in Fig. 1, neonatal treatment with $100 \mu$ g oestradiol benzoate or $50 \mu \mathrm{g} \mathrm{CI-628}$ alone resulted in a decreased availability of cytoplasmic oestrogen binding sites in the uterus of the prepubertal rat. While nafoxidine alone did not affect later expression of oestrogen receptor protein in the uterus, the subsequent neonatal exposure to oestradiol at $24 \mathrm{~h}$ after nafoxidine injection resulted in a prepubertal expression of oestrogen receptor production similar to that seen in response to the neonatal exposure to oestradiol benzoate alone.

Figure 2 indicates that oestrogen stimulation of glucose oxidation in the prepubertal rat uterus was significantly reduced from control levels $(P<0.025)$ in animals which had been treated neonatally with oestradiol benzoate or nafoxidine alone or with oestradiol benzoate in combination with either of the antioestrogens. The mean value for each of these treatments did not differ from the average of the unstimulated control group which was injected with saline instead of oestradiol $3 \mathrm{~h}$ before assay. The metabolic response to oestradiol in the treatment groups resembled that observed in rats which had been neonatally ovariectomized. Thus, only neonatal treatment with CI-628 alone did not produce an adverse effect on this uterine response.

An experiment to determine the extent to which the antioestrogens $\mathrm{Cl}-628$ or nafoxidine deplete specific cytoplasmic oestrogen binding site availability $24 \mathrm{~h}$ after neonatal injection was also conducted (Table 1). Nafoxidine given as a single $50 \mu \mathrm{g}$ injection $24 \mathrm{~h}$ before assay for cytoplasmic oestrogen binding by the charcoal-dextran procedure reduced the measured estimation of control uterine cytosolic receptor by $81 \cdot 5 \%$. The same dose of CI-628 produced a $72 \cdot 8 \%$ depletion of cytoplasmic oestrogen binding, sites in the uterus.

The ovarian weights of prepubertal rats treated neonatally with steroid hormone ester and/or antioestrogen are given in Table 2 . A significant reduction $(P<0.05)$ in ovarian weights was evident for all treatment groups when compared to the peanut oil vehicle-injected controls. This reduction in prepubertal ovarian weight in treatment groups is largely coincident with reduced oestrogen binding sites in the uterus and the impairment of oestrogen-stimulated glucose metabolism. However, only the relationship between the relative magnitude of ovarian weight reduction as a consequence of the different neonatal treatments and the degree to which uterine glucose metabolism is compromised exhibits a significant correlation coefficient $(P<0.05)$. 


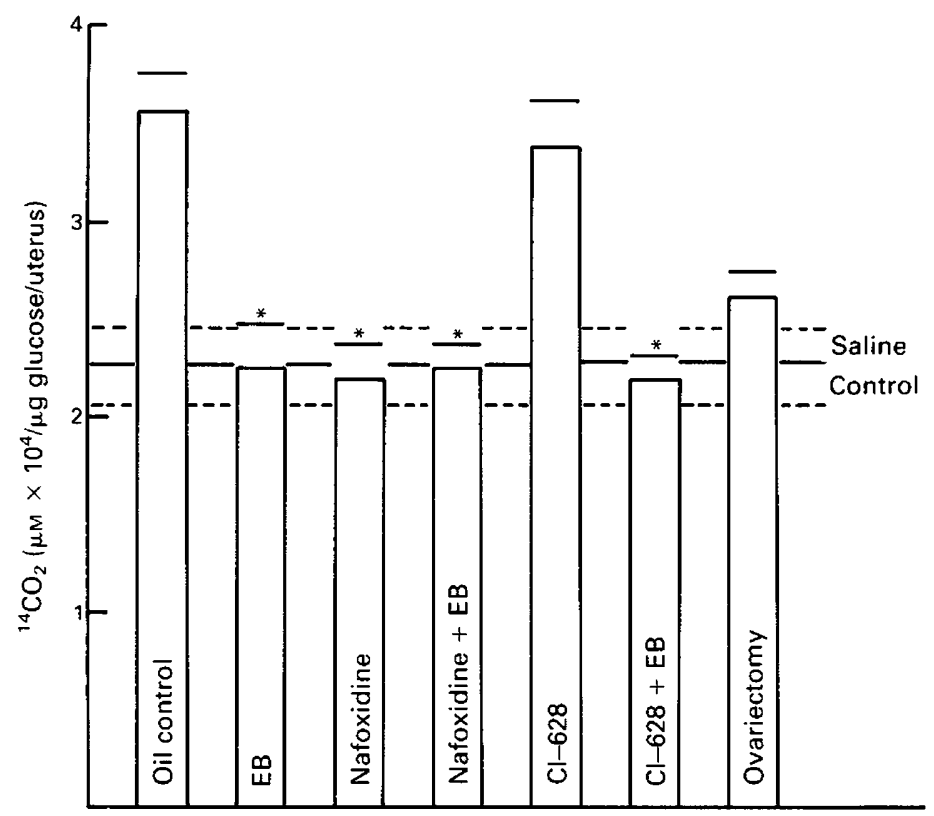

Fig. 2. In-vitro determination of oestradiol-stimulated glucose metabolism in uteri of prepubertal rats neonatally ovariectomized or treated with oestradiol benzoate (EB) or antioestrogen (nafoxidine or CI-628). Each value is the mean \pm s.e.m. of at least 8 separate experiments. The broken lines represent the upper and lower s.e.m. limits for the mean basal rate of glucose oxidation (saline-injected controls). ${ }^{*} P<0.025$ compared with control value (Dunnett's T).

Table 1. Depletion of cytoplasmic oestrogen binding sites in uteri of prepubertal rats injected with antioestrogen

\begin{tabular}{lccc}
\hline Treatment & $\begin{array}{c}\text { No.of } \\
\text { rats }\end{array}$ & $\begin{array}{c}{\left[{ }^{3} \mathrm{H}\right] \text { Oestradiol }} \\
\text { bound per uterus } \\
\text { after injection } \\
(\mathrm{pmol} / 24 \mathrm{~h})\end{array}$ & $\begin{array}{c}\% \text { Depletion of } \\
\text { cytoplasmic } \\
\text { oestrogen binding } \\
\text { sites }\end{array}$ \\
\hline Peanut oil & 8 & $1.51 \pm 0.20$ & 0.0 \\
$50 \mu \mathrm{g}$ CI-628 & 8 & $0.41 \pm 0.09$ & 72.8 \\
$50 \mu$ g Nafoxidine & 8 & $0.28 \pm 0.03$ & 81.5 \\
\hline
\end{tabular}

Values are mean \pm s.e.m.

The effect of neonatal treatment with oestradiol benzoate alone or $24 \mathrm{~h}$ after prior treatment with nafoxidine or CI-628 upon oestrogenic induction of uterine actomyosin in adulthood is presented in Table 3. Uteri from animals treated neonatally with oestradiol benzoate exhibited a significant reduction $(P<0.05)$ from control actomyosin production. In rats in which both antioestrogens had been administered $24 \mathrm{~h}$ before neonatal oestradiol benzoate injection, intermediate organ concentrations of actomyosin were evident after oestrogen treatment in adulthood. However, when uterine actomyosin content was expressed on the basis of total uterine protein, the relative actomyosin content in animals treated neonatally with antioestrogen plus oestradiol benzoate is similar to that seen in control animals. 
Table 2. Relationship between ovarian weight (mean \pm s.e.m.) and the availability of uterine cytoplasmic oestrogen binding sites and uterine glucose metabolism in animals treated neonatally with oestradiol benzoate (EB), nafoxidine (NAF) and CI-628

\begin{tabular}{lcccc}
\hline Neonatal treatment & No. of rats & Ovarian wt $(\mathrm{mg})$ & $\mathrm{A}^{*}$ & $\mathrm{~B}^{*}$ \\
\hline Oil (control) & 26 & $13 \cdot 5 \pm 0.6$ & & \\
$100 \mu \mathrm{g} \mathrm{EB}$ & 22 & $6 \cdot 2 \pm 0 \cdot 4^{* *}$ & + & + \\
$50 \mu \mathrm{g} \mathrm{NAF}+100 \mu \mathrm{g} \mathrm{EB} \dagger$ & 17 & $7 \cdot 6 \pm 0 \cdot 3^{* *}$ & + & + \\
$50 \mu \mathrm{g} \mathrm{NAF}$ & 16 & $7 \cdot 0 \pm 0.3^{* *}$ & - & + \\
$50 \mu \mathrm{g} \mathrm{CI}-628+100 \mu \mathrm{g} \mathrm{EB} \dagger$ & 18 & $5 \cdot 6 \pm 0.4^{* *}$ & + & + \\
$50 \mu \mathrm{g} \mathrm{CI}-628$ & 16 & $8 \cdot 5 \pm 0.4^{* *}$ & + & - \\
\hline
\end{tabular}

${ }^{*} \mathrm{~A}=$ relationship between ovarian weight and oestrogen binding sites; correlation coefficient $=0.51 ; \mathrm{B}=$ relationship between ovarian weight and glucose metabolism; correlation coefficient $=0.87(P<0.05)$. A positive $(+)$ relationship indicates that both ovarian weight and the experimental uterine parameter varied in a parallel fashion from control. A negative ( - ) relationship indicates that only the ovarian weight or the experimental uterine parameter alone is significantly different from control.

$\dagger$ Injections given $24 \mathrm{~h}$ apart.

${ }^{* *} P<0.005$ (Dunnett's T test) compared with control.

Table 3. Uterine actomyosin content in adult rats treated neonatally with oestradiol benzoate (EB) or with the combination of nafoxidine (NAF) or CI-628 plus EB

\begin{tabular}{lccc}
\hline & \multicolumn{2}{c}{ Actomyosin } \\
\cline { 3 - 4 } Treatment & No. of rats & $\mathrm{mg} / \mathrm{g}$ uterus & $\mathrm{mg} / \mathrm{mg}$ total protein \\
\hline Oil control & 7 & $3 \cdot 25 \pm 0 \cdot 24$ & $0.074 \pm 0.007$ \\
$100 \mu \mathrm{g} \mathrm{EB}$ & 7 & $1 \cdot 11 \pm 0 \cdot 15^{*}$ & $0.030 \pm 0.005^{*}$ \\
$50 \mu \mathrm{g} \mathrm{NAF}+100 \mu \mathrm{g} \mathrm{EB} \dagger$ & 4 & $2 \cdot 10 \pm 0 \cdot 82$ & $0.068 \pm 0.034$ \\
$50 \mu \mathrm{g} \mathrm{Cl}-628+100 \mu \mathrm{g} \mathrm{EB} \dagger$ & 5 & $2 \cdot 32 \pm 0 \cdot 54$ & $0.072 \pm 0.020$ \\
\hline
\end{tabular}

Values are mean \pm s.e.m.

†Injections spaced $24 \mathrm{~h}$ apart.

${ }^{*} P<0.05$ (Neuman-Keuls statistics) compared with control value.

\section{Discussion}

It is evident from the results that neonatal exposure to the antioestrogens CI-628 or nafoxidine does not allay the effects of subsequent neonatal oestradiol benzoate treatment upon later prepubertal expression of uterine oestrogen receptor protein or metabolic enzymes of the phosphogluconate oxidative pathway. However, while the antagonist properties of these antioestrogens are not effective on these two measures, the two antioestrogens are acting quite differently with respect to their agonist properties. Nafoxidine simulates oestrogen in its disruptive effect upon the development of the uterine glucose metabolic pathway, while CI-628 simulates oestradiol benzoate action on the impairment of cytoplasmic oestrogen binding sites in the prepubertal rat uterus. This differential effect may be a reflection of variable cell specific agonist properties of antioestrogens (Markaverich et al., 1981; Clark et al., 1982; Dohler et al., 1984). 
The ovarian weight reduction observed in the prepubertal rat treated neonatally with steroid ester or antioestrogen probably represents feedback suppression of gonadotrophin secretion due to the introduction of exogenous agonist (Clark et al., 1973; Gellert et al., 1977; Campbell, 1980). Additionally, previous reports have demonstrated a causal relationship between prepubertal ovarian weights and uterine sensitivity to oestrogen-stimulated growth or glucose oxidation (Campbell, 1980; Campbell \& Modlin, 1987). In this regard, neonatal treatment with CI-628, unlike nafoxidine, is without effect upon development of the uterine phosphogluconate oxidative pathway, and CI-628 treatment does not result in the reduction of prepubertal ovarian weights to the same extent as do other treatments. Furthermore, the production of oestrogen receptor protein is clearly not dependent upon endogenous oestrogen since neonatal ovariectomy does not affect this measure (Clark \& Gorski, 1970). The effect of neonatal exposure to oestradiol benzoate or CI628 upon the reduction of oestrogen binding sites in the prepubertal uterus probably, therefore, represents a direct tissue response to the agonist. Hence, neonatal exposure to agonist may 'turn off' oestrogen receptor production and decrease the production of normal levels of enzymes in the metabolic pathway for glucose oxidation for entirely different reasons. The early impairments in the development of the reproductive tract observed in this study are consistent with the induction of reproductive anomalies in adulthood as a result of perinatal exposure to antioestrogens such as clomiphene, nafoxidine and tamoxifen (Gellert et al., 1971; Clark \& McCormack, 1977; Chamness et al., 1979; McCormack \& Clark, 1979; Clark et al., 1982; Dohler et al., 1986).

Neonatal treatment with nafoxidine or CI-628 before neonatal injection of oestradiol benzoate $24 \mathrm{~h}$ later, however, did prevent the oestradiol-induced reduction of oestrogen-stimulated production of actomyosin in adult rat uteri. The hyperoestrogenization of the uterus due to antioestrogen exposure occurs principally in the endometrium: the antioestrogens do not affect the myometrium acutely or developmentally on histological or biochemical bases while oestradiol affects luminal epithelium, stroma and myometrium (Markaverich et al., 1981; Clark et al., 1982). Therefore, in tissue in which both antioestrogens lack agonist properties, their antagonist actions appear to prevail.

Although we observed some apparent cytoplasmic oestrogen binding sites remaining after neonatal antioestrogen injection, these residual binding sites may not be available for subsequent nuclear translocation (Williams \& Gorski, 1974). Moreover, depletion of all cytoplasmic receptors does not appear to be a prerequisite for the antagonistic action of the antioestrogens (Hayes et al., 1981). However, the mechanism of action of the antioestrogens upon reproductive tract development is complicated by the presence of specific antioestrogen binding sites distinct from oestrogen receptors in a number of tissues in the rat, including the uterus and ovary (Sutherland et al., 1980; Winneker \& Clark, 1983; Sudo et al., 1983). Indeed, it has been proposed that antioestrogen interaction with such binding sites may alter biological potencies and pharmacokinetics to produce differential effects (Sudo et al., 1983). Clearly, the possibility that antioestrogen binding sites may mediate some of the effects of the antioestrogens needs to be examined.

Furthermore, previous reports have indicated that antioestrogens are uterotrophic in the neonatal rat (Clark et al., 1982; Clark \& Guthrie, 1983). The results described herein also confirm the prominent agonist properties of the antioestrogens CI-628 and nafoxidine on neonatal reproductive tract tissues. Each antioestrogen produces prepubertal reproductive deficits that differentially mimic those produced by oestradiol benzoate, while neither antioestrogen blocks the oestradiol-induced impairment which is not produced when the antioestrogen is administered alone to the neonatal rat.

Since some antioestrogens are being used clinically to induce ovulation in anovulatory women and to inhibit breast cancer, the dual potential of these compounds needs to be recognized to avoid unexpected and perhaps undesirable side effects. The results reported in this work therefore reaffirm and extend the caution raised previously in using these drugs during pregnancy and/or the perinatal period (Clark \& McCormack, 1977; McCormack \& Clark, 1979; Chamness et al., 1979). In addition to the structural and functional reproductive anomalies previously observed in 
adulthood, it is evident from this study that early, prepubertal alterations at the molecular level also occur as a consequence of exposure to antioestrogens early in life.

\section{References}

Anderson, J.N., Peck, E.J., Jr \& Clark, J.H. (1973) nuclear receptor-estrogen complex: relationship between concentration and early uterotrophic responses. Endocrinology 92, 1488-1495.

Camphell, P.S. (1980) Impaired prepubertal uterine responsivity after neonatal exposure to steroid hormone esters. J. exp. Zool. 214, 345-353.

Campbell, P.S. \& Modlin, P.S. (1987) Uterine glucose metabolism in the prepubertal rat treated neonatally with androgen, estrogen, and antihormones. Experientia 43, 309-310.

Chamness, G.C., Bannayan, G.A., Landry, L.A., Jr., Sheridan, P.J. \& McGuire, W.L. (1979) Abnormal reproductive development in rats after neonatally administered antiestrogen (tamoxifen). Biol. Reprod. 21, 1087-1090.

Clark, J.H. \& Gorski, J. (1970) Ontogeny of the estrogen receptor during early uterine development. Science, N.Y. 169, 76-78.

Clark, J.H. \& Guthrie, S.C. (1983) The estrogenic effects of clomiphene during the neonatal period in the rat. J. Steroid Biochem. 18, 513-517.

Clark, J.H. \& McCormack, S.A. (1977) Clomid or nafoxidine administered to neonatal rats causes reproductive tract abnormalities. Science, N.Y. 197, $164-165$

Clark, J.H., Anderson, J.N. \& Peck, E.J., Jr (1973) Estrogen receptor-anti-estrogen complex: atypical binding by uterine nuclei and effects on uterine growth. Steroids 22, 707-718.

Clark, J.H., Guthrie, S.C. \& McCormack, S.A. (1982) Neonatal stimulation of the uterus by clomiphene, tamoxifen and nafoxidine: relationship to the development of reproductive tract abnormalities. In Hormones and Cancer, pp. 87-98. Ed. W. W. Leavitt. Plenum Publishing Corporation, New York.

Dohler, K.D., Strivastava, S.S., Shryne, J.E., Jarzab, B., Sipos, A. \& Gorski, R. A. (1984) Differentiation of the sexually dimorphic nucleus in the preoptic area of the rat brain is inhibited by postnatal treatment with an estrogen antagonist. Neuroendocrinology 38, 297-301.

Dohler, K.D., Coquelin, A., Davis, F. Hines, M., Shryne, J.E., Sickmoller, P.M., Jarzab, B. \& Gorski, R.A. (1986) Pre- and postnatal influence of an estrogen antagonist and an androgen antagonist on differentiation of the sexually dimorphic nucleus of the preoptic area in male and female rats. Neuroendocrinology 42, 443-448.

Gellert, R.J., Bakke, J.L. \& Lawrence, N.L. (1971) Persistent estrus and altered estrogen sensitivity in rats treated neonatally with clomiphene citrate. Fert. Steril. 22, 244-250.

Gellert, R.J., Lewis, J. \& Petra, P.H. (1977) Neonatal treatment with sex steroids: relationship between the uterotrophic response and the estrogen "receptor" in prepubertal rats. Endocrinology 100, 520-528.

Hayes, J.R., Rorke, E.A., Katzenellenbogen, B.S., Robertson, D.W. \& Katzenellenbogen, J.A. (1981) Biological potency and uterine estrogen receptor interactions of the metabolites of the antiestrogens CI628 and U23,469. Endocrinology 108, 164-172.

Korenman, S.G. (1968) Radio-ligand binding assay of specific estrogens, using a soluble uterine macromolecule. J. clin. Endocr. Metab. 28, 127-130.

Lowry, O.H., Rosebrough, N.J., Farr, A.L. \& Randall, R.J. (1951) Protein measurement with the Folin phenol reagent. J. biol. Chem. 193, 265-275.

Markaverich, B.M., Upchurch, S., McCormack, S.A., Glasser, S.R. \& Clark, J.H. (1981) Differential stimulation of uterine cells by nafoxidine and clomiphene: relationship between nuclear estrogen receptors and type II estrogen binding sites and cellular growth Biol. Reprod. 24, 171-181.

McCormack, S. \& Clark, J.H. (1979) Clomid administration to pregnant rats causes abnormalities of the reproductive tract in offspring and mothers. Science, N.Y. 204, 629-631.

Needham, D.M. \& Williams, J.M. (1963) Proteins of the uterine contractile mechanism. Biochem. J. 89, 552-561.

Nicolette, J.A. \& Gorski, J. (1964) Effect of estradiol on glucose $-\mathrm{U}-\mathrm{C}^{14}$ metabolism in the rat uterus. Archs Biochem. Biophys. 107, 279-283.

Sudo, K., Monsma, F.J., Jr. \& Katzenellenbogen, B.S. (1983) Antiestrogen-binding sites distinct from the estrogen receptor: subcellular localization, ligand specificity, and distribution in tissues of the rat. Endocrinology 112, 425-434.

Sutherland, R.L., Murphy, L.C., Foo, M.S., Green, M.D., Whybourne, A.M. \& Krozowski, Z.S. (1980) High affinity antioestrogen binding site distinct from the oestrogen receptor. Nature, Lond. 288, 273-275.

Williams, D. \& Gorski, J. (1974) Equilibrium binding of estradiol by uterine cell suspensions and whole uteri in vitro. Biochemistry, N.Y. 13, 5537-5542.

Winneker, R.C. \& Clark, J.H. (1983) Estrogenic stimulation of the antiestrogen specific binding site in rat uterus and liver. Endocrinology 112, 1910-1915.

Received 11 August 1987 